\section{The role of mouse models in translational cancer research: present and future directions}

\author{
Manuela Porru, Carlo Leonetti \\ UOSD SAFU, Department of Research, \\ Diagnosis and Innovative Technologies, \\ Traslational Research Area, Regina \\ Elena National Cancer Institute, Rome, \\ Italy
}

\begin{abstract}
A major issue in the research for new anticancer therapeutics is represented by the low number of new compounds approved for clinical use in comparison to the good success rate reported in preclinical studies. This high attrition rate could be attributed to many factors including the unsatisfactory predictive value of experiments performed in animals. To this purpose, general opinions suggest that classical models as murine tumors and xenografts do not mimic the complexity of human cancer diseases and that the use and integration of different relevant mouse models could improve the efficiency of the drug development process. In this review, we overview the present state of research in the field of mouse models for cancer and describe the advantages and limitations of the different models. Finally, strategies for improving the predictive value of animal experiments will be also discussed.
\end{abstract}

\section{Introduction}

Animal research has played an important role in the advances of medical science in the 20th century providing invaluable insights in the pathogenesis of most diseases and in pharmacological research. In particular, the discovery and the development of the top 20 prescribed essential drugs including the nonsteroidal antiinflammatory Aspirin, Paracetamol used for pain relief and reducing fever or Metformin the antidiabetic drug, were all made possible by the contribution of animal experiments. A paradigmatic example of this is diabetes research. There is no doubt that patients have benefited from the use of animal models in the discovery of insulin and other treatments for the different types of this pathology. ${ }^{1}$ Nevertheless, the power of animal experiments in predict- ing the clinical outcome remains particularly controversial. One of the major concerns, despite the rate of successful preclinical experiments, is that the percentage of authorized agents is very low. In fact, $85 \%$ of early clinical trials for novel drugs fail and of those that survive through to phase III, only half are eventually approved for clinical use. ${ }^{2}$ To this purpose, the largest proportion of these failures occurs in trials for cancer where only $5 \%$ of agents having anticancer activity in preclinical development are approved. As a result an impressive

number of very promising preclinical studies requiring much effort in time and money and loss of animal lives did not translate from the bench to the bedside. ${ }^{3}$ This strong discrepancy between preclinical efficacy and clinical response could be attributed to numerous factors such as the complexity of cancer disease and the need of integrating biomarker response to improve patient selection in clinical trials for cancer precision medicine. ${ }^{4}$ Besides these important issues, animal modeling undoubtedly represents a major challenge in improving the translation efficiency. The most frequently used tool in preclinical anticancer drug studies is in vitro studies carried out on established human cell lines combined with implanting the same cells under the skin or intramuscularly in immunocompromised mice. While this strategy is valid as an indicator of possible clinical activity and is able to identify a candidate drug evaluated for having scientific merit in justifying further development, the predictive value for clinical outcome is limited. ${ }^{5}$ In fact, a number of features associated with their use must be acknowledged, such as: i) the blood supply and neovascularization is provided by the host and is derived from murine derived stroma cells; ii) tumors are implanted in a tissue compartment different from the original site; iii) these models do not recapitulate the heterogeneity of tumors in patients; iv) metastases are not very common; v) the lack of a fully functioning immune system. Unfortunately, at this moment no single mouse cancer model capturing the different aspects of the human disease and predictive of the clinical outcome, exists. On the contrary, there are a number of advanced preclinical models, which should be utilized and integrated during specific stages of the development process following a well-defined aim of the research, thus helping to improve our ability to translate preclinical data into clinical success. $^{6}$
Correspondence: Carlo Leonetti, Regina Elena National Cancer Institute, Via Elio Chianesi 53, Rome, 00144 Italy.

Phone +390652662534 ; fax +390652662579 .

E-mail: carlo.leonetti@ifo.gov.it

Key words: Experimental model, Mice, Patient-derived xenografts, Genetically engineered mouse models, Orthotopic tumors.

Acknowledgments: we are grateful to Adele Petricca for secretarial assistance and to Tania Merlino for the English revision. This work was supported by a grant from Italian Association for Cancer Research AIRC IG \#18637.

Contributions: MP and CL contributed to the writing of the manuscript.

Received for publication: 25 January 2017.

Revision received: 20 May 2017.

Accepted for publication: 18 July 2017.

This work is licensed under a Creative Commons Attribution 4.0 License (by-nc 4.0).

CC Copyright M. Porru and C. Leonetti 2017 Licensee PAGEPress, Italy

Translational Medicine Reports 2017; 1:6598 doi:10.4081/tmr.6598

\section{Patient derived xenografts}

Patient derived xenografts (PDXs) are obtained by the implantation of fresh human tumor fragments following a small incision made in the subcutis of immunosuppressed mice, usually in SCID, NODSCID or NOD-SCID NOD-SCID IL2RGamma null (NSG) mice. ${ }^{7}$ Tumors are maintained by passaging fragments directly from mouse to mouse and then expanded for the evaluation of anticancer efficacy of treatments. Usually the time for engraftment is between 2 and 4 months and failure of engraftment should not be ascertained until at least 6 months and beyond. ${ }^{8}$ PDXs from most tumor types have been successfully established for Non-Small Cell Lung Cancer, ${ }^{9}$ breast cancer, ${ }^{10}$ melanoma, ${ }^{11}$ head and neck tumors, ${ }^{12}$ metastatic colon cancer, ${ }^{13}$ bladder cancer ${ }^{14}$ and ovarian cancer. ${ }^{15}$ One of the main features of PDX models is the maintenance of the cellular and histological structure of the original tumour including critical stromal elements, which provide sustenance under periods of extensive growth. ${ }^{8}$ Moreover, cytogenetic analysis of tumors from PDXs revealed strong preservation of the overall genomic and gene expression profile of the 
corresponding patient tumours..$^{8,10,16}$ Interestingly, in regards to the predictive value of PDXs for clinical outcome, several studies have demonstrated a concordance between the patient response to chemotherapy and PDXs of the same tumor histotype. For example, human breast cancer PDXs responded to Trastuzumab-based combination similarly to patients ${ }^{17}$ and colonrectal cancer PDXs mimicked the sensitivity/resistance to Cetuximab of patients. ${ }^{13}$

These results demonstrated that PDXs represent a powerful experimental tool for the development of anticancer therapeutics which could help us to identify the most effective therapy for the single cancer of a patient or subgroup of patients. In this context, one interesting idea may be to use a PDX as an avatar model, by treating PDXs obtained from a patient enrolled in a clinical trial with the same therapy administered to the patient, thus permitting to identify biomarkers of sensitivity or resistance to treatments.

Alternatively, as reported by Misale et al., ${ }^{18}$ PDXs could be useful in selecting effective therapeutic strategies for secondline treatment. In fact, these authors demonstrated in PDXs derived from patients eliciting resistance to anti-EGFR inhibitors, that a dual blockade of EGFR and MEK with Cetuximab/Pimasertib combination induced a complete response in mice, thus tumours remained undetectable for more than six months. In this context, Bousquet et al., ${ }^{19}$ showed that PDXs from a patient with an invasive triple-negative breast carcinoma which elicited a disease relapse after chemotherapy and radiation, were sensitive to Paclitaxel plus Cetuximab therapy. Following these results in PDXs, the patient was treated with Paclitaxel plus Cetuximab as second-line treatment and after 3 months almost a complete metabolic response was observed associated with a time to progression longer than previous lines of treatment.

\section{Orthotopic and dissemination disease model}

One major drawback of classical xenografts and PDXs is that cells or tissue fragments are implanted subcutis (s.c.) or intramuscular (i.m.) but these in general do not correspond to the primary site of human cancer. Moreover, stromal and micro environmental spatial and paracrine interactions with host non-cancerous cells and tissues which have a key role in modulating tumor drug sensitivity, are absent. ${ }^{20}$ At the same time, after s.c. or i.m implantation, tumours fail to progress or to metastasize and therefore do not retain all the patterns of the disease course observed in patients. To address this issue, orthotopic models of cancer have been established over the last two decades by implanting cell lines and more recently tumor fragments through developing the so-called patient-derived orthotopic xenografts (PDOXs). The Hoffman and Fidler groups were pioneers in this research area showing that the activity of chemotherapeutics, observed in syngeneic and immune-compromised orthotopic models, better reflect the clinical activity of drugs such as Cisplatin, Mitomycin $\mathrm{C}$ and Doxorubicin than ectopic models. ${ }^{21,22}$ Based on the ability of orthotopic models to reconstitute the tumor microenviroment and reproduce malignant progression, in terms of localized and distal metastatic spread of the primary tumor, they represent a great opportunity to develop new drugs able to target specific molecules involved in these processes..$^{23,24}$ One major limitation of the orthotopic models is that the cell lines are artificially selected from the original tumors which have adapted to growth outside the natural tumor microenvironment, resulting in genetic changes that are distinct from those imposed on tumors in patients. Moreover, the inter- and intra-tumor heterogeneity that characterises primary human tumors is loss. ${ }^{6}$ One strategy adopted in addressing these key points in the science of preclinical modeling is the use of PDOXs whereby tumour fragments from patients are directly implanted in mice in the orthotopic sites of origin. Many interesting examples of PDOXs have been reported demonstrating these models' ability to reproduce human disease. In particular, the establishment of breast or pancreatic carcinoma tumors into the mammary glands or in the pancreas of mice maintain clinical features of original tumors as it is shown by the majority of mice that developed metastases corresponding to patient metastatic sites, such as lymph nodes, lungs, bone and peritoneum in the case of breast cancer or peritoneal and liver metastasis for pancreas PDOXs. ${ }^{25,26}$ The potential of PDOXs to better predict the response of tumors to anticancer therapy compared to subcutaneous models has also been highlighted by a recent paper where HER-2 expressing cervical carcinoma PDOXs were evaluated. ${ }^{27}$ These authors showed that PDOXs recapitulated the original cervical cancer of the patient both in terms of primary tumor and as peritoneal disseminated disease. Most interestingly, therapy with the benzamide histone deactylase inhibitor, Entinostat was effective against the metastasis while no response to the treatment was observed in the primary tumors of PDOXs and in the subcutaneous PDXs model of the same tumor, thus highlighting the importance of using PDOXs in the development of antimetastatic therapies. Recently, imaging modalities for in situ assessment of primary tumor growth and metastatic dissemination have been developed using different systems. In particular, the use of Fluorodeoxyglucosepositron emission tomography (FDG-PET) and an approach which combines micro PET and F(ab')2 fragments of the fullyhuman anti-EGFR monoclonal antibody, panitumumab, have been

successfully evaluated. ${ }^{28,29}$ Moreover, a fluorescently-labelled chimeric anti-CEA antibody was able to detect the primary tumor and the residual mass after the surgical resection in whole colon cancer PDOXs. ${ }^{30}$

\section{Genetically engineered mouse models}

The growing knowledge of human genetics has enabled the use of a wide array of genetically engineered mouse models (GEMMs) which have permitted to unveil or better understand the role of many genes in normal physiological or malignant processes including cancer. These models are established by modifying genes in different ways and by introducing these genetic modifications into the germ line or into specific tissue or cell types through conditional knockout strategy. ${ }^{31}$ GEMMs are able to mimic changes responsible for the development and progression of cancer and are also capable of recapitulating different types of human cancers thus contributing to identifying mutational events which act as genetic drivers and modifiers, as well as modulating the response to therapies. ${ }^{32}$ One of the main features that distinguish GEMMs from ectopic or orthotopic xenografts is represented by the maintenance of the tumor-stroma tissue or organ microenviroment of an immuno-competent host. $^{31}$ Therefore, the role of immune response or immunosurveillance in the response to chemotherapeutics should be more thoroughly studied in GEMMs than in classical immunodeficient mice. A good example of GEMMs application in the study of key regulators of inflammatory and immune response is the work of Meylan et al..$^{33}$ They used a mouse model of lung cancer with defined mutations in Kras and p53, which showed that activation 
of the NFKB pathway is a critical event in lung tumorigenesis. Interestingly, the authors demonstrated that treatment with $\mathrm{NF} \kappa \mathrm{B}$ antagonists led to significant impairment of tumor development. This effect was associated with an inflammatory response as observed by the recruitment of inflammatory cells to tumors.

A major hurdle in fighting cancer is the need to cure the numerous number of diseases we call cancer. In fact, we now know that cancer develops in many different genetic subtypes and this diversity determines the response or resistance to treatments. From this perspective, GEMM may be useful in reproducing the different forms of cancer of the same histotype in vivo to identify effective therapies. A paradigm is represented by the studies on acute promyelocytic leukemia (APL) which has many subtypes, genetically heterogeneous and shows different responses to therapy. ${ }^{34}$ At the same time APL is a rare disease, representing approximately $10 \%$ of all myeloid leukemias and consequently, given that patients have to be stratified based on the genetic patterns, the number of patients to enroll for clinical trials is frequently never enough to give statistical significance. To overcome these limitations, Pandolfi's group ${ }^{35}$ reproduced, in large cohort of mice, various human fusion genes and biological and pathological characteristics of human diseases as well as validate GEMMs for the chemotherapeutic response. Importantly, their study permitted to discover new treatment modalities for APL leading to the approval of the histone deacetylase inhibitor, Suberoylanilide- hydroxamic acid (SAHA), for clinical use. This strategy has been applied also in the solid tumor context developing a series of GEMMs representing different patient populations with the same cancer. They also demonstrated, as in the case the prostate cancer, the role of specific genetic alterations in the response to androgendeprivation therapy together with the close correlation between the response of patients and GEMMs to the same therapy. ${ }^{36}$ Despite the obvious advantages and results obtained by using GEMMs, this model has a number of limitations including the length of time and the high costs necessary to develop and maintain a sufficient number of mice to produce statistically significant data. This is also a consequence of the heterogeneity of GEMMs in tumor development with regard to frequency, latency and growth characteristics, an aspect which poses significant problems when several experiment groups are evaluated. ${ }^{31}$ Moreover, GEMMs do not completely model extensive genetic alterations, tumor heterogeneity nor different aspects of disease progression and metastasis observed in human cancers. ${ }^{37}$ The use of imaging modalities are essential for assessing tumor growth and dissemination as well as for evaluating the efficacy of therapies. The costs for carrying out these analyses need consistent investment initially which largely limit the large-scale use of the GEMMs model.

It is for these reasons that, it has been suggested to not use GEMMs in the initial stages of drug development but rather to apply them later, in order to optimize lead compounds in clinically relevant GEMMs to effectively support the translational drug process. $^{6}$

\section{Humanized mouse models}

Recent advances in our understanding of the interplay between cancer cells and the surrounding stromal tissue highlight the important role of the microenvironment in cancer growth, dissemination of metastasis and response to therapy. In fact, tumors release chemokines which influence the growth of stroma cells that at the same time produce factors affecting tumor growth. Moreover, the inhibition of such interactions producing continuous T-cell activation, led to the development of novel anticancer immunotherapeutics such as ipilimumab, nivolumab and pembrolizumab. ${ }^{38}$

Given that for the efficacy of these therapies is crucial in a functional immune system, the use of classical immunodepressed mice for drug preclinical development is very limited thus making research for more suitable experimental models vital. To this purpose, since the discovery of NOD-SCID immunodeficient mice bearing mutations in the IL2 receptor common gamma chain (IL2rgnull) in the early 2000s, different types of humanized mice have been developed from severely immunodeficient mouse strains such as NOD/Shi-scid-IL2rgnull (NOG), ${ }^{39}$ NOD/LtSz-sciIL2rgnull (NSG) ${ }^{40}$ BALB/c Rag2null IL2rgnull, ${ }^{41}$ partially reconstituting the human immune system. In early

Table 1. The main features of the most advanced mouse models for cancer biology and drug discovery studies.

\begin{tabular}{|c|c|c|c|}
\hline Type & Mouse species & Advantages & Limitations \\
\hline Patient-derived xenografts & Immunodeficient & $\begin{array}{l}\text { - Maintain tumor } \\
\text { heterogeneity, stromal components, histological } \\
\text { and molecular characteristics of patient tumors } \\
\text { - Easy propagation for testing new drugs and } \\
\text { combinations }\end{array}$ & $\begin{array}{l}\text { - Low take rate and long } \\
\text { time frame required for engraftment } \\
\text { - Lack of metastatic spread (s.c. implant) } \\
\text { - Fail to replicate interactions with } \\
\text { human microenviroment }\end{array}$ \\
\hline $\begin{array}{l}\text { Orthotopic and } \\
\text { dissemination models }\end{array}$ & $\begin{array}{l}\text { Immunodeficient and } \\
\text { immunocompetent }\end{array}$ & $\begin{array}{l}\text { - Assessment of drug } \\
\text { activity on primary tumor and metastases } \\
\text { - Tumor stroma- microenviroment interaction } \\
\text { is maintained (immunocompetent mice) }\end{array}$ & $\begin{array}{l}\text { - High variability in } \\
\text { primary tumor growth and metastatic spread } \\
\text { - Need of advanced real-time imaging systems } \\
\text { - Very skilled personnel is required }\end{array}$ \\
\hline $\begin{array}{l}\text { Genetically engineered } \\
\text { mouse models }\end{array}$ & Immunocompetent & $\begin{array}{l}\text { - Well defined genetic driver of tumor } \\
\text { development and progression } \\
\text { - Maintenance of tumor stroma-microenviroment } \\
\text { interactions } \\
\text { - Presence of intact immunosystem }\end{array}$ & $\begin{array}{l}\text { - Partial modeling of genetic alterations } \\
\text { observed in human cancer } \\
\text { - Time of tumor development is heterogeneous } \\
\text { - Metastatic pattern do not mimic human cancers }\end{array}$ \\
\hline Humanized mouse models & Immunodeficient & $\begin{array}{l}\text { - Similarities with human cancer in tumor structure, } \\
\text { metastasis and signaling } \\
\text { Growth of human tumor in a context of functional } \\
\text { human immunosystem } \\
\text { - Development of new immunotherapeutics }\end{array}$ & $\begin{array}{l}\text { - Variability in tumor of } \\
\text { stroma and immunocell infiltration } \\
\text { - Presence of immunoreactive T cells } \\
\text { - Incomplete B-cell differentiation } \\
\text { and activation of antigen-specific response }\end{array}$ \\
\hline
\end{tabular}


studies, humanized mice were derived by the direct injection of human peripheral blood but more recently are generated by the engraftment in mice of CD34+ human hematopoietic stem and precursor cells (HSPC) obtained by fetal cord blood. These mice developed a human immune system with differentiated and functional $\mathrm{T}$ and $B$ cells, macrophages, and other cells capable of interacting with xenograft tumor tissues..$^{40}$ The implantation of tumor cells in these mice permitted to highlight a specific immuno-response stimulated by the growth of the tumor. ${ }^{42,43}$ Very recently, Morton et al. ${ }^{43}$ reported that by adding purified Tat- MYC and Tat-Bcl2 fusion proteins to cultured HSPCs the number of CD34+ cells markedly expanded, while transduced HSPCs retained the ability to differentiate into either myeloid or lymphoid cells, thus recapitulating both lineages of the human immune system. Interestingly, in head and neck PDXs, the relationship between the tumor and immune system was similar to the one found in the patient in whom the tumor originated was observed. In particular, the authors observed that the stroma surrounding the tumors had human $\mathrm{T}$ and $\mathrm{B}$ cell infiltration, the stromal microenvironment was reprogrammed, with alteration of gene expressions governing the extracellular matrix, the immune response, and the epithelial-mesenchymal transition. ${ }^{44}$

Importantly, humanized mice may be useful not only for studying human diseases but also for discovering more effective anticancer therapies. Even though we are still in the early stages of this type of technology, there are several recently published studies showing the utility of humanized mice in the development of immunomodulatory anticancer agents. In particular, Chang et al. ${ }^{45}$ demonstrated the antitumor efficacy of monoclonal antibodies (mAbs) targeting carbonic anhydrase (CA) IX, a surface-expressed protein, in an orthotopic model of renal cell carcinoma (RCC) that was implanted in mice humanized by injection of allogeneic human peripheral blood mononuclear cells. Antitumor activity of anti-CAIX mAbs correlated with the ability of mediating human immune response in vivo including tumor infiltration of NK cells and activation of $\mathrm{T}$ cells. Interestingly, by using humanized mice, Monk et al. ${ }^{46}$ are developing an integrative approach for the treatment of ovarian cancer based on the combination of motolimod, a novel Toll-like receptor 8 (TLR8) agonist that stimulates robust innate immune responses with pegylated liposomal doxorubicin (PLD), a chemotherapeutic agent that induces immunogenic cell death. This study performed on healthy human volunteers, non-human primates, humanized mice reconstituted with human $\mathrm{CD} 34+$ cells and cancer patients demonstrated the efficacy of this combination therapy. In particular, the experiments performed in humanized mice implanted with ovarian cancer cells proved very useful since they demonstrated that the potent immunomodulation observed in mice following combination therapy was due to tumor-infiltrating monocytes and $\mathrm{T}$ cells which was associated with significant tumor control. Experiments in mice were the determining factor for scheduling treatment and on these bases a phase 2 study was initiated.

The major limitations of humanized mice and possible strategies to improve the potential of this preclinical model have been highlighted in a Morton et al.'s review. ${ }^{44}$ In this review, one of the main key points raised was in regard to the eventual presence of mature xenoreactive $\mathrm{T}$ cells in humanized mice created by the injection of purified CD34+ cells. In this case, $\mathrm{T}$ cells could recognize tumor cells as a foreign body and possibly kill them as it was observed that tumors implanted in humanized mice grow more slowly than tumors implanted on the same non-humanized mice strain. ${ }^{44}$ This causes concern when antitumor efficacy of new compounds is evaluated; therefore, future studies should aim to create humanized mice in which HSPCs and the tumor are derived from the same patient.

\section{Conclusions}

For many reasons mice represent the model organism of choice for basic cancer research and for drug discovery as its genome is very similar to the human genome and share common physiological and pathological processes. Moreover, given that mice are well known for their small size and short generation time it permits researchers to use a number of mice, which makes results statistically significant. Since the first studies

reporting on the use of in vivo murine leukemia models for drug efficacy in the $1950 \mathrm{~s}^{47}$ and the discovery of the nude mutation on chromosome 11 of athymic mice lacking T- lymphocytes in $1966,{ }^{48}$ many efforts have been devoted to the development of mice models in predicting the response of chemotherapeutics in humans. Today, there is a very large panel of advanced mouse models which can mimic the complexity and the heterogene- ity of human cancers, specific stages of tumor initiation, progression and metastatization, the role of the vasculature, the microenviroment and immune cells. It is essential to recognize that each model has advantages and limitations (Table 1) and consider that no single model is able to capture all the different aspects of tumor growth, characteristics and treatment approaches. ${ }^{6,48}$ For example, GEMMs and PDX models can be regarded as complementary and not interchangeable since PDX models are generated in immunocompromised mice, while GEMM models are immune competent and at the same time PDX models are genetically heterogeneous, while GEMMs are genetically identifiable by their engineered genetic alterations. ${ }^{49}$ In conclusion, the use and integration of different models which better recapitulate the clinical situation and are able to answer specific experimental questions seem to be essential in the drug discovery process and in improving our translational ability, which could reduce attrition rate between preclinical studies and clinical application.

\section{References}

1. Rees DA. Animal models of diabetes mellitus. Diabet Med 2005;22:359-70.

2. Ledford H. Translational research: 4 ways to fix the clinical trial. Nature 2011;477:526-8.

3. Hutchinson L, Kirk R. High drug attrition rates-where are we going wrong? Nat Rev Clin Oncol 2011;8:189-90.

4. Rubin EH, Gilliland DG. Drug development and clinical trials-the path to an approved cancer drug. Nat Rev Clin Oncol 2012;9:215-22.

5. Sausville EA, Burger AM. Contributions of human tumor xenografts to anticancer drug development. Cancer Res 2006;66:3351-4.

6. Ruggeri BA, Camp F, Miknyoczki S. Animal models of disease: pre-clinical animal models of cancer and their applications and utility in drug discovery. Biochem Pharmacol 2014;87:15061.

7. Pompili L, Porru M, Caruso C, et al. Patient-derived xenografts: a relevant preclinical model for drug development. J Exp Clin Cancer Res 2016;35: 189.

8. Tentler JJ, Tan AC, Weekes CD, et al. Patient-derived tumour xenografts as models for oncology drug development. Nat Rev Clin Oncol 2012;9:33850 . 
9. John T, Kohler D, Pintilie M, et al. The ability to form primary tumor xenografts is predictive of increased risk of disease recurrence in early-stage non-small cell lung cancer. Clin Cancer Res 2011;17:134-41.

10. Reyal F, Guyader C, Decraene C, et al. Molecular profiling of patient-derived breast cancer xenografts. Breast Cancer Res 2012;14:R11.

11. Némati F, Sastre-Garau X, Laurent C, et al. Establishment and characterization of a panel of human uveal melanoma xenografts derived from primary and/or metastatic tumors. Clin Cancer Res 2010;16:2352-62.

12.Klinghammer K, Raguse JD, Plath T, et al. A comprehensively characterized large panel of head and neck cancer patient-derived xenografts identifies the mTOR inhibitor everolimus as potential new treatment option. Int $\mathrm{J}$ Cancer 2015;136:2940-8.

13.Bertotti A, Migliardi G, Galimi F, et al. A molecularly annotated platform of patient- derived xenografts ("xenopatients") identifies HER2 as an effective therapeutic target in cetuximab-resistant colorectal cancer. Cancer Discov 2011;1:508-23.

14. Bernardo C, Costa C, Sousa N, et al. Patient-derived bladder cancer xenografts: a systematic review. Transl Res 2015;166:324-31.

15. Topp MD, Hartley L, Cook M, et al. Molecular correlates of platinum response in human high-grade serous ovarian cancer patient-derived xenografts. Mol Oncol 2014;8:65668.

16. Zhang XC, Zhang J, Li M, et al. Establishment of patient-derived nonsmall cell lung cancer xenograft models with genetic aberrations within EGFR, KRAS and FGFR1: useful tools for preclinical studies of targeted therapies. J Transl Med 2013;11:168.

17. Marangoni E, Vincent-Salomon A, Auger N, et al. A new model of patient tumor- derived breast cancer xenografts for preclinical assays. Clin Cancer Res 2007;13:3989-98.

18. Misale S, Bozic I, Tong J, et al. Vertical suppression of the EGFR pathway prevents onset of resistance in colorectal cancers. Nat Commun 2015;6:8305.

19. Bousquet G, Feugeas JP, Ferreira I, et al. Individual xenograft as a personalized therapeutic resort for women with metastatic triple-negative breast carcinoma. Breast Cancer Res 2014;16:401.

20. McMillin DW, Negri JM, Mitsiades CS. The role of tumor-stromal interactions in modifying drug response: challenges and opportunities. Nat Rev Drug Discov 2013;12:217-28.

21. Kuo TH, Kubota T, Watanabe M, et al. Site-specific chemosensitivity of human small-cell lung carcinoma growing orthotopically compared to subcutaneously in SCID mice: the importance of orthotopic models to obtain relevant drug evaluation data. Anticancer Res 1993;13:627-30.

22. Fidler IJ, Wilmanns C, Staroselsky A, et al. Modulations of tumor cell response to chemotherapy by the organ environment. Cancer Metastasis Rev 1994:13:209-22.

23. Smith PA, Merritt D, Barr L, et al. An orthotopic model of metastatic nasopharyngeal carcinoma and its application in elucidating a therapeutic target that inhibits metastasis. Genes Cancer 2011;2:1023-33.

24. Loi M, Di Paolo D, Becherin P, et al. The use of the orthotopic model to validate antivascular therapies for cancer. Int J Dev Biol 2011;55:547-55.

25. De Rose YS, Wang G, Lin YC, et al. Tumor grafts derived from women with breast cancer authentically reflect tumor pathology, growth, metastasis and disease outcomes. Nat Med 2011;17:1514-20.

26. Walters DM, Stokes JB, Adair SJ, et al. Clinical, molecular and genetic validation of a murine orthotopicxenograft model of pancreatic adenocarcinoma using fresh human specimens. PLoS One 2013;8:e77065.

27. Hiroshima Y, Maawy A, Zhang Y, et al. Patient-derived mouse models of cancer need to be orthotopic in order to evaluate targeted anti-metastatic therapy. Oncotarget 2016;7:71696-702.

28. Braekeveldt N, Wigerup C, Gisselsson $\mathrm{D}$, et al. Neuroblastoma patientderived orthotopic xenografts retain metastatic patterns and geno- and phenotypes of patient tumours. Int J Cancer 2015;136:E252-61.

29. Boyle AJ, Cao PJ, Hedley DW, et al. MicroPET/CT imaging of patientderived pancreatic cancer xenografts implanted subcutaneously or orthotopically in NOD- scid mice using (64)CuNOTA-panitumumab $F\left(a b^{\prime}\right) 2$ fragments. Nucl Med Biol 2015;42:71-7.

30. Hiroshima Y, Maawy A, Sato S, et al. Hand-held high-resolution fluorescence imaging system for fluorescence-guided surgery of patient and cell-line pancreatic tumors growing orthotopically in nude mice. J Surg Res 2014;187:510-7.

31. Kucherlapati R. Genetically modified mouse models for biomarker discov- ery and preclinical drug testing. Clin Cancer Res 2012;18:625-30.

32. Lunardi A, Nardella C, Clohessy JG, Pandolfi PP. Of model pets and cancer models: an introduction to mouse models of cancer. Cold Spring Harb Protoc 2014;2014:17-31.

33. Meylan E, Dooley AL, Feldser DM, et al. Requirement for NFkappaBsignalling in a mouse model of lung adenocarcinoma. Nature 2009; 462:104-7.

34. Piazza F, Gurrieri C, Pandolfi PP. The theory of APL. Oncogene 2001;20:7216-22.

35. Nardella C, Lunardi A, Patnaik A, et al. The APL paradigm and the "co-clinical trial" project. Cancer Discov 2011;1:108-16.

36.Lunardi A, Ala U, Epping MT, et al. A co-clinical approach identifies mechanisms and potential therapies for androgen deprivation resistance in prostate cancer. Nat Genet 2013;45:747-55.

37. Clohessy JG, Pandolfi PP. Mouse hospital and co-clinical trial projectfrom bench to bedside. Nat Rev Clin Oncol 2015;12:491-8.

38. Khalil DN, Smith EL, Brentjens RJ, Wolchok JD. The future of cancer treatment: immunomodulation, CARs and combination immunotherapy. Nat Rev Clin Oncol 2016;13:273-90.

39. Hiramatsu H, Nishikomori R, Heike $\mathrm{T}$, et al. Complete reconstitution of human lymphocytes from cord blood CD34p cells using the NOD/ SCID/gammacnull mice model. Blood 2003;102:873-80.

40. Shultz LD, Lyons BL, Burzenski LM, et al. Human lymphoid and myeloid cell development in NOD/LtSz-scid IL2R gamma null mice engrafted with mobilized human hematopoietic stem cells. J Immunol 2005;174:6477-89.

41. Traggiai E, Chicha L, Mazzucchelli L, et al. Development of a human adaptive immune system in cord blood cell-transplanted mice. Science 2004;304:104-7.

42. Wege AK, Ernst W, Eckl J, et al. Humanized tumor mice-A new model to study and manipulate the immune response in advanced cancer therapy. Int J Cancer 2011;129:2194-206.

43. Morton JJ, Bird G, Keysar SB, et al. XactMice: humanizing mouse bone marrow enables microenvironment reconstitution in a patient-derived xenograft model of head and neck cancer. Oncogene 2016;35:290-300.

44. Morton JJ, Bird G, Refaeli Y, Jimeno A. Humanized Mouse Xenograft Models: Narrowing the Tumor- 
Microenvironment Gap. Cancer Res 2016;76:6153-8.

45. Chang D-K, Moniz RJ, Xu Z, et al. Human anti-CAIX antibodies mediate immune cell inhibition of renal cell carcinoma in vitro and in a humanized mouse model in vivo. Mol Cancer 2015;14:119.

46. Monk BJ, Facciabene A, Brady WE, et al. Integrative development of a
TLR8 agonist for ovarian cancer chemo-immunotherapy. Clin Cancer Res 2017;23:1955-66.

47. Kirschbaum A, Geisse NC, Sister TJ, Meyer LM. Effect of certain folic acid antagonists on transplanted myeloid and lymphoid leukemias of the $\mathrm{F}$ strain of mice. Cancer Res 1950;10:762-8.

48. Wartha K, Herting F, Hasmann M. Fit- for purpose use of mouse models to improve predictivity of cancer therapeutics evaluation. Pharmacol Ther 2014;142:351-61.

49. Abate-Shen C, Pandolfi PP. Effective utilization and appropriate selection of genetically engineered mouse models for translational integration of mouse and human trials. Cold Spring Harb Protoc 2013;2013:11. 